\title{
Blood pressure standards for very low birthweight infants during the first day of life
}

\author{
J Lee, V S Rajadurai, K W Tan
}

\begin{abstract}
Blood pressures during the first day of life were measured prospectively in 61 very low birthweight infants using umbilical or peripheral arterial lines. Video recordings of real time waveforms were reviewed. Blood pressure correlated linearly with birthweight and gestation. Comparison with available standards showed that infants weighing under $800 \mathrm{~g}$ had lower acceptable mean arterial pressure (MAP). The lower limits of MAP for infants between 26 to 32 weeks of gestation were numerically similar to the gestational ages.
\end{abstract}

(Arch Dis Child Fetal Neonatal Ed 1999;81:F168-F170)

Keywords: mean arterial blood pressure; very low birthweight

Large numbers of very low birthweight (VLBW) infants (birthweight under $1500 \mathrm{~g}$ ) receive treatment for supposed hypotension during the first day of life. ${ }^{1}$ Blood pressure norms for VLBW infants are scarce, but the values in the study by Versmold et al are commonly cited. ${ }^{2}$ A few factors might reduce their validity, including the small number (16) of extremely low birthweight infants (ELBW, birth weight under $1000 \mathrm{~g}$ ), the exclusion of infants given volume expanders when they might have had normal blood pressure, and the assumption of linearity of blood pressure vs birthweight for a wide weight range (610-4200 g). We had noticed that in daily practice some ELBW infants had lower limits of blood pressure below those cited by Versmold et al but no signs of hypotension. We therefore decided to embark on this study using larger number of infants, taking more frequent, valid readings and including only VLBW infants.

\section{Methods}

This was a prospective, observational study. All VLBW infants with an umbilical or peripheral arterial line were recruited. Exclusion criteria were inotropic support, culture confirmed sepsis, Apgar scores of $<5$ at 5 minutes, intraventricular haemorrhage (IVH) grades III and IV during the first week, twin-twin transfusion and major congenital malformations.

Blood pressure was measured in the standard manner using high umbilical lines or radial/posterior tibial lines. Only nondampened readings were valid. Respiratory waveforms were simultaneously monitored. When infants cried or moved, these waveforms became irregular and readings during these times were not taken. Readings were also excluded five minutes after crying or noxious stimuli and one hour after infusion of volume expanders. Real time blood pressure, respiratory rate, heart rate, and transcutaneous oxygen saturation waveforms were videotaped and subsequently reviewed. Blood pressure readings were taken every five minutes and an average value was obtained for each hour of life. Mean values were then obtained for every infant for two periods: the first and second 12 hours of life, respectively. During the period of the study the decision to start inotropes was based on signs of hypoperfusion such as pallor and poor capillary return, and not on blood pressure readings alone.

Linear regression analysis was used to obtain standards of blood pressure against birthweight and gestational age. Individual regression prediction lines with $95 \%$ confidence limits were obtained. Multiple linear regression was applied to evaluate the influence of variables on blood pressure. Birthweight and gestational age were analysed separately to eliminate the effect of collinearity. Univariate analysis of variance was used to compare blood pressure between appropriate for gestational age and small for gestational age infants while controlling for birthweight and gestational age, respectively. The significance level was taken at 0.05.

The study was approved by the hospital's ethics committee.

\section{Results}

Blood pressure values from 61 VLBW infants were studied over 13 months from February 1996 to March 1997. There were 28 (45.9\%) ELBW infants and $34(55.7 \%)$ infants with gestational ages of 28 weeks and under. Blood pressure data were available for analysis in 56 infants for the entire 24 hour period. Three infants had blood pressure data sufficient for analysis only during the period 13 to 24 hours after birth and two infants had data only during the first 12 hours of life. A total of 30072 data points were used for analysis (10024 each for systolic, diastolic, and mean blood pressures). This meant that every infant had a blood pressure value included about every 8.8 minutes.

The mean birthweight was $996 \mathrm{~g}$, with a standard deviation of $281 \mathrm{~g}$, and the mean gestational age was 28.0 weeks (SD 2.8 weeks). Forty two infants $(69 \%)$ had umbilical lines. Mean Apgar score at 5 minutes was 7.7 (SD 1.3). Sixty two per cent received antenatal steroids and $44 \%$ were treated with indomethacin after 24 hours of life for a haemodynamically significant patent ductus arteriosus (PDA). Most infants had mild to moderate lung disease. Forty three per cent received sur- 


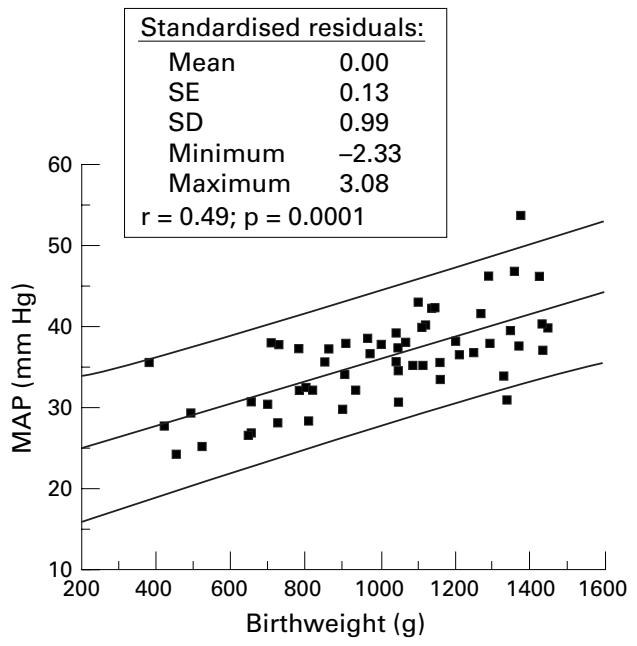

Figure 1 Average MAP 13 to 24 hours after birth compared with birthweight.

factant. At 24 hours of life, only $52 \%$ were receiving intermittent mandatory ventilation (IMV) with a mean airway pressure of $6.0 \mathrm{~cm}$ $\mathrm{H}_{2} \mathrm{O}$ (SD $1.9 \mathrm{~cm} \mathrm{H}_{2} \mathrm{O}$ ), 33\% were receiving continuous positive airway pressure (CPAP), and $15 \%$ head box ventilation. Twenty three per cent of the infants were small for gestational age (birthweight below the tenth centile for their gestational age). Eighteen per cent received volume expanders once during the first 12 hours of life.

Scatter plots of systolic, mean, and diastolic blood pressures against birthweight and gestational age were constructed for both time periods, resulting in 12 graphs altogether (fig 1). There were good linear relations between blood pressure and birthweight, and between blood pressure and gestational age. The linear correlation coefficients $(\mathrm{r})$ ranged from 0.44 to 0.74 .

Tables 1 and 2 show the lower 95\% confidence limits of MAP derived from the linear regression graphs for the various birthweights and gestational ages during both time periods. These represent the conventional definition for hypotension. Blood pressures were higher 13 to 24 hours after birth compared with the first 12 hour period. The lower limits of MAP were numerically similar to the gestational age at 26 to 32 weeks for the second time period. Compared with Versmold's results, infants weighing under $800 \mathrm{~g}$ in our study had lower acceptable MAP (table 1).

For a similar gestational age, small for gestational age infants had lower blood pres-

Table 1 Lower 95\% CI for MAP ( $\mathrm{mm} \mathrm{Hg}$ ) vs birthweight (g)

\begin{tabular}{llllllllllll}
\hline Birthweight & 500 & 600 & 700 & 800 & 900 & 1000 & 1100 & 1200 & 1300 & 1400 & 1500 \\
\hline $0-12$ hours & 20 & 21 & 22 & 23 & 24 & 26 & 27 & 28 & 29 & 30 & 31 \\
& (NA) & $(24)$ & $(24)$ & $(25)$ & $(25)$ & $(26)$ & $(26)$ & $(27)$ & $(27)$ & $(28)$ & $(28)$ \\
$13-24$ hours & 21 & 22 & 23 & 25 & 26 & 28 & 29 & 30 & 32 & 33 & 34 \\
\hline
\end{tabular}

( ) refers to the study by Versmold et al

Table 2 Lower 95\% CI for MAP ( $\mathrm{mm} \mathrm{Hg}$ ) vs gestational age (weeks)

\begin{tabular}{llllllllllllll}
\hline Gestational age & 23 & 24 & 25 & 26 & 27 & 28 & 29 & 30 & 31 & 32 & 33 & 34 & 35 \\
\hline $0-12$ hours & 20 & 21 & 22 & 23 & 25 & 26 & 27 & 28 & 29 & 30 & 31 & 32 & 33 \\
$13-24$ hours & 20 & 22 & 23 & 25 & 27 & 28 & 29 & 30 & 32 & 33 & 35 & 36 & 37 \\
\hline
\end{tabular}

sure compared with appropriate for gestational age infants (small: mean 32.98 (SE 1.10) mm $\mathrm{Hg}$; appropriate: mean 36.83 (SE 0.57) $\mathrm{mm}$ $\mathrm{Hg} ; \mathrm{p}=0.003$ ). For a similar birthweight, small for gestational age and appropriate for gestational age infants had similar blood pressure (small: mean 37.34 (SE 1.16) $\mathrm{mm}$ $\mathrm{Hg}$; appropriate: mean 35.60 (SE 0.61 ) $\mathrm{mm}$ $\mathrm{Hg} ; \mathrm{p}=0.193)$. As an illustration, during the second 12 hour period, a 28 week old small for gestational age infant had an MAP at the lower 95\% confidence limit of $22 \mathrm{~mm} \mathrm{Hg}$, compared with an appropriate for gestational age counterpart at $30 \mathrm{~mm} \mathrm{Hg}$ - a difference of $8 \mathrm{~mm}$ $\mathrm{Hg}$. On the other hand, an $800 \mathrm{~g}$ infant who was small for gestational age, had a value of 28 $\mathrm{mm} \mathrm{Hg}$ vs $29.5 \mathrm{~mm} \mathrm{Hg}$ for his/her appropriate for gestational age counterpart-a difference of only $1.5 \mathrm{~mm} \mathrm{Hg}$.

Possible confounders of blood pressure (5 minute Apgar score, antenatal steroids, surfactant, volume expanders and PDA) were looked at for both time periods, but they did not have a significant effect (multiple linear regression, $\mathrm{p}>0.05$ ) with one exception (volume expanders during the second 12 hour period, $\mathrm{p}=0.02$ ). With over 20 comparisons, this might easily have been due to chance.

\section{Discussion}

Our method of collecting blood pressure data differed from normal. We videotaped real time blood pressure and respiratory waveforms continuously so that when these were reviewed later, frequent readings could be taken and inappropriate readings excluded, thus reducing within patient variation. The results of our study showed that for infants under $800 \mathrm{~g}$, acceptable blood pressures were lower than those described by Versmold. Treatment is perhaps not necessary for a "low" blood pressure value alone unless there are coexisting signs of hypoperfusion, such as poor capillary return, oliguria, and metabolic acidosis. Unnecessary infusion of colloids or crystalloids can contribute to morbidity.

Superimposing our linear regression line for MAP during the first 12 hours on Versmold's, the relation between blood pressure and birthweight might not have been linear for the range of birthweight between $610 \mathrm{~g}$ and $4220 \mathrm{~g}$, which was the birthweight range in Versmold's study. This non-linearity may be partly responsible for the difference between the two studies. Further research will be needed to prove this.

Questions are always posed as to how to define a normal population among the VLBW infants admitted to neonatal intensive care. In this study very ill infants and those receiving inotropes were excluded. Most had mild to moderate lung disease. Only half were still receiving IMV at the end of day 1 with very low mean airway pressure, thus there would not have been much effect of increased intrathoracic pressure on blood pressure. Infants with clinically significant IVH were excluded in view of the possible cause and effect relation between hypotension and IVH. ${ }^{3}$ In addition, none had periventricular leucomalacia, a lesion that might be caused by hypotension during 
the first few days after birth. ${ }^{4}$ This would strengthen our assertion that none of the babies included here was hypotensive. Volume expansion did not modify blood pressure throughout the study period. Hence it was reasonable to infer that the 11 babies given volume expansion during the first few hours of life were normotensive and that they should be included. Blood pressure was similar in appropriate and small for gestational age infants of identical birthweight, but blood pressure was lower in small, than in appropriate, for gestational age infants of identical gestational age. In daily practice, when dealing with a small for gestational age infant it is thus reasonable to use birthweight related standards.

First day blood pressure measured directly has been studied by others as well but their methodology differed widely from that used in our study. Watkins et al collected retrospective hourly data from VLBW infants. ${ }^{5}$ Their reported MAP were much higher than ours for those babies below $800 \mathrm{~g}$ at birth. The British Association of Perinatal Medicine and Royal College of Physicians defines hypotension as an MAP of less than an infant's gestational age in completed weeks. ${ }^{6}$ This is remarkably similar to our results for infants between 26 and 32 weeks of gestation. It is a useful rule of thumb unless an infant is small for gestational age where even lower blood pressure values were acceptable.
In summary, we had obtained blood pressure standards using data from non-hypotensive VLBW infants with conditions frequently seen in neonatal intensive care, and these differed from those described by others with respect to the tiny ELBW infants. We conclude that it may not be necessary to treat an infant for a low blood pressure value that is greater than the lower limits of normal unless $\mathrm{s} / \mathrm{h}$ e has other signs of hypoperfusion.

This study was funded by the National Medical Research Council, Singapore. We thank Stephanie Chong for her help in statistical analysis.

1 Hope P. Pump up the volume? The routine early use of colloid in very preterm infants. Arch Dis Child Fetal Neonatal Ed 1998;78:F163-5.

2 Versmold HT, Kitterman JA, Phibbs RH, Gregory GA, Tooley WH. Aortic blood pressure during the first 12 hours of life in infants with birth weight 610 to 4,220 grams. Pediatrics 1981;67:607-13.

3 Miall-Allen VM, De Vries LS, Whitelaw AGL. Mean arterial blood pressure and neonatal cerebral lesions. Arch Dis Child 1987;62:1068-9.

4 Volpe JJ. Brain injury in the premature infant: neuropathology, clinical aspects, pathogenesis and prevention. Clin Perinatol 1997;24:567-87.

5 Watkins AMC, West CR, Cooke RWI. Blood pressure and cerebral hemorrhage and ischemia in very low birth weight infants. Early Hum Dev 1989;19:103-10.

6 Joint Working Party of British Association of Perinatal Medicine and the Research Unit of the Royal College of Physicians. Development of audit measures and guidelines for good practice in the management of neonatal for good practice in the management of neonatal
respiratory distress syndrome. Arch Dis Child 1992; 67:1221-7. 